\title{
LIDIA D'AGOSTINO : UNA HISTORIA DEDICADA A LA MOTRICIDAD OROFACIAL
}

Belkis David Parra Reyes ${ }^{21}$

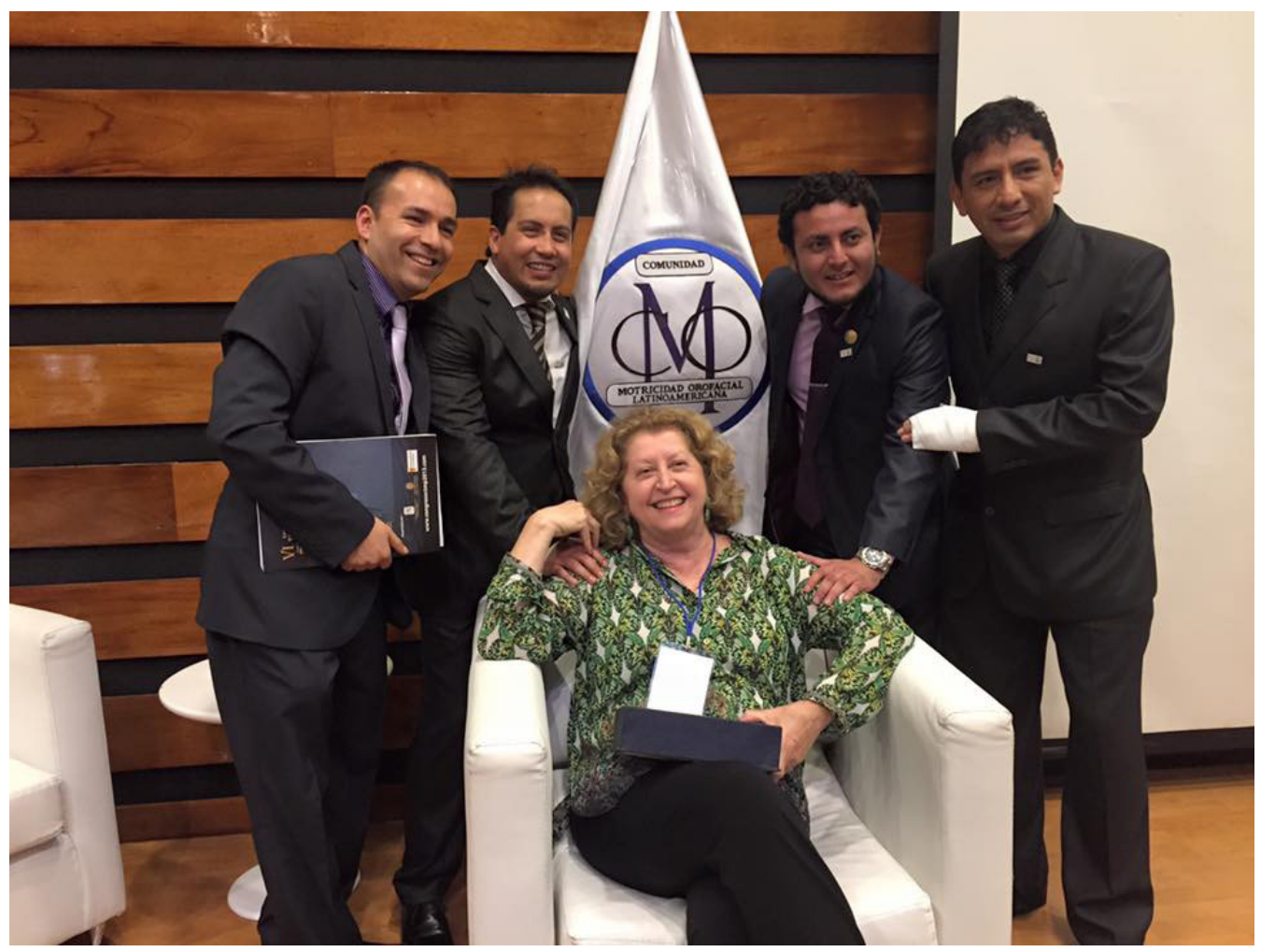

La historia de Lidia D'Agostino se inicia en el año de 1967 cuando partió de una pequeña ciudad del interior de São Paulo, Matão, para estudiar Fonoaudiología en la Pontificia Universidad de São Paulo - PUC-SP. La opción por el curso fue después conocer el trabajo de las Fonoaudiólogas Teresinha Del Papa, Lilia Salomão y Ana María en el Hospital de Servicio Público de São Paulo, por sugerencia de un primo médico de aquel hospital.

El curso de Fonoaudiología estaba en la sexta clase y no era reconocido por el Ministerio de Educación -MEC-. La facultad ofrecía un certificado de finalización del curso. En presencia de tal realidad muchas colegas desistieron del curso con miedo de un futuro incierto y fue en ese momento que se hizo una promesa a si misma "luchar mucho y honrar la profesión elegida para el que dependiera de mí, estaría en lo correcto".

21.Doctor en Ciencias de la Educación por la Universidad Nacional de Educación. Magister en Neurociencias por la Facultad de Medicina de la Universidad Nacional Mayor de San Marcos. Especialista en Motricidad Orofacial otorgado por CPAL - CEFAC. Diplomado en Docencia Universitaria/Docencia en Educación superior por la UNE. Licenciado en Tecnología Médica en la Especialidad de Terapia de Lenguaje por la Universidad Nacional Federico Villarreal. Terapeuta de Lenguaje en Rehabilitación Orofacial en síndromes Neurológicos en el Hospital Nacional Guillermo Almenara I. - EsSALUD. Docente en pre-grado y postgrado de diversos cursos especializados sobre Terapia de Lenguaje en la UNFV, UNMSM y UAP. Miembro de la International Association Of Orofacial Myology (IAOM), de la Academia Latinoamericana de Disfunciones Estomatognáticas (ALDE), Miembro fundador de la Academia de Neurociencias del Perú (ANP), de la Asociación Peruana para el Estudio del Dolor (APED), Miembro fundador de la Comunidad de Motricidad Orofacial Latinoamericana (CMOL), Miembro del cuerpo editorial en la calidad de editor de la revista científica de Medicina de Rehabilitación del Departamento de Medicina de Rehabilitación del Servicio de otras Discapacidades y Minusvalías del HNGAI, de la revista CoDaS (Brasil), columnista de la Revista de Ciencia y Cultura ANRA, Miembro de la Comisión de Investigación del Hospital Nacional Guillermo Almenara Irigoyen, Miembro del Instituto de Investigación de la FTM y del comité consultivo interno como docente investigativo invitado por el Vicerrectorado de Investigación de la UNFV. Director y editor de la revista digital de motricidad Orofacial "RevMOf". Director científico de la Comunidad de Motricidad Orofacial Latinoamericana (CMOL). 
A pesar de los escasos recursos bibliográficos y la falta de profesionales con amplia experiencia para orientarnos, contábamos con profesores empeñados en hacer de la Fonoaudiología una profesión del futuro entre los cuales destaco el añorado Dr. Mauro Spinelli, Dr. Alfredo Tabith Jr, Prof. Fanny Amado, Prof. Magnani; Dr. Orozimbo Alves; Prof. Isidoro Blinkstai.

Finalizado el curso en 1969 la invitación de una de las profesoras de Logopedia, Profesora Fanny Amado, fue trabajar en la clínica CLIDEME de la Asociación de Padres y Amigos Excepcionales (APAE) que iniciaba sus actividades de Estudio, enseñanza e investigación en Deficiencia Mental bajo la dirección del psiquiatra infantil Dr. Stanislau Krinsky. El trabajo era interdisciplinar y renombrados profesionales del área crearon el protocolo de atención y fueron encargados de formar nuevos profesionales recién formados y por lo tanto sin vicios de trabajo "a su imagen y perfección". Durante un año pasamos por todas las áreas aprendiendo como actuaban y más intensamente en el área específica pues en las palabras del Dr Krinsky "actuar en equipo interdisciplinario es conocer mucho de su área y lo suficiente de las demás para saber interpretar los exámenes y hablar el mismo lenguaje".

Al pasar por la Genética su interés por los síndromes fue despertando y al constatar los efectos de la dismorfia en la parte funcional y de la hipotonía orofacial presente, busco desarrollar ejercicios miofuncionales para reducir los problemas orofaciales. Los síndromes cráneofaciales más complejos, Síndrome de Apert, de Treacher Collins entre otras, les angustiaban, pues los portadores eran erróneamente considerados deficientes mentales por su aspecto y la actuación del fono quedaba muy limitada por la malformación ósea. En la APAE aprendió a actuar en equipo adquiriendo nuevos conocimientos que le permitirán ver al paciente como un TODO, analizar los problemas y buscar soluciones.

En 1978 al sentir una perdida gradual de la masticación, paso por consulta con un equipo de cirugía cráneofacial del Instituto de Recuperación Humana del Hospital São Joaquim de la Beneficencia Portuguesa y el procedimiento indicado fue cirugía ortognática para corrección de la oclusión.

El director del equipo, Dr. Jorge Miguel Psillakis, al saber de su profesión le hace una propuesta: no cobraria la cirurgia con la condición y el compromiso en retornar durante un año después del pós operatório para estudios pues ocurría que los pacientes operados satisfechos con los resultados no retornaban para el seguimiento y después de un año lo hacían con queja de recidivas.

En los reportes pos operatorio relata los problemas funcionales, posturales y musculares remanentes, relataba también como solucionaba esos problemas con ejercicios específicos. Por mis relatos el Dr. Psillakis concluye que los problemas funcionales interferían en la estabilidad de las cirugías y que era necesario el trabajo del fono y así fui invitada a participar del equipo para desarrollar métodos de evaluación y tratamiento relacionados a las cirugías cráneofaciales.

La experiencia adquirida en la APAE con el estudio de los síndromes y las largas discusiones de casos con los cirujanos fue la sumatoria para el nuevo aprendizaje pues el área cráneofacial era aún inexplorada por la Fonoaudiología y no había bibliografía específica disponible. Gracias al Dr. Psillakis que con su mente abierta, años por delante de sus compañeros, y a los demás profesionales del equipo, Dra. Vera Cardim, Dr. Fausto Viterbo, Dr. Matteó entre otros con sus enseñanzas le fue posible desarrollar el trabajo. La experiencia fue muy gratificante pues los problemas óseos que limitaban el trabajo miofuncional con los pacientes de la APAE eran reducidos y hasta solucionados con la cirugía cráneofacial.

"Mi objetivo dentro de la Cirugía Cráneofacial fue divulgar el trabajo del fonoaudiólogo junto a los equipos de cirujanos craneofaciales en los cursos y congresos del área advirtiéndoles sobre la importancia de los problemas funcionales involucrados en las cirugías y en los eventos de la Fonoaudiología para conocimiento de la nueva área de actuación". 
"En ese período tuve participación en libros de cirugía plástica cráneofacial con diez capítulos de fonoaudiología. Fiel a su antigua promesa llego a enfrentar con conferencias la resistencia de 500 cirujanos en congresos donde ella era la única fonoaudióloga. Mi determinación rindió frutos, hoy reconocidos cirujanos reconocen la importancia del trabajo del fonoaudiólogo y sus equipos cuentan con el profesional".

En el año de 1980 fue la primera fonoaudióloga contratada por la administración directa de la SECRETARIA DE LA SALUD DE LA PREFECTURA DE SÃO PAULO, Un hospital de tercera línea dedicado al estudio y diagnóstico de patologías complejas, para actuar en el equipo ambulatorio de Especialidades del Hospital Infantil Niño Jesús con la responsabilidad de demostrar la importancia del trabajo del fonoaudiólogo y volver conocida la profesión y así abrir nuevas oportunidades de trabajo para los colegas de la profesión.

Con ese objetivo llegó a atender 50 pacientes en terapia semanal, tocando de porta en puerta de los consultorios médicos para discutir los casos y posteriormente mostrando los resultados.

En Hospital vio la oportunidad de aumentar sus conocimientos con lo aprendido de las patologías pediátricas y aplicar la experiencia adquirida anteriormente y así ayudo a formar un equipo interdisciplinario de Fisuras Labio palatinas con profesionales de diez especialidades.

En el año de 1986 con el cierre de las actividades del Instituto de Recuperación Humana, fue invitada a participar del equipo del Dr. José Marcos Mélega en el Instituto de Cirugía Plástica Santa Cruz donde actúa hasta la presente fecha en la atención de las fisuras labio palatinas en colaboración con la clínica de Trauma y Prótesis Maxilofacial de la Facultad de Odontología de la USP-SP.

A partir de 2009 participa del equipo de la Dra. Vera Cardim en el Centro de Plástica Avanzada - Hospital San Joaquin de la Beneficencia Portuguesa, en la atención de casos complejos de malformaciones craneofaciales.

Para Lídia: En los cuarenta y dos años de trabajo su objetivo siempre fue honrar a la Fonoaudiología demostrando la importancia en la nueva área de actuación, fue un trabajo arduo en el enfrentamiento de las dificultades por la falta de recursos técnicos y del desconocimiento de la profesión, pero también trajo muchas alegrías y el orgullo de poder decir : "SOY FONOAUDIÓLOGA" !
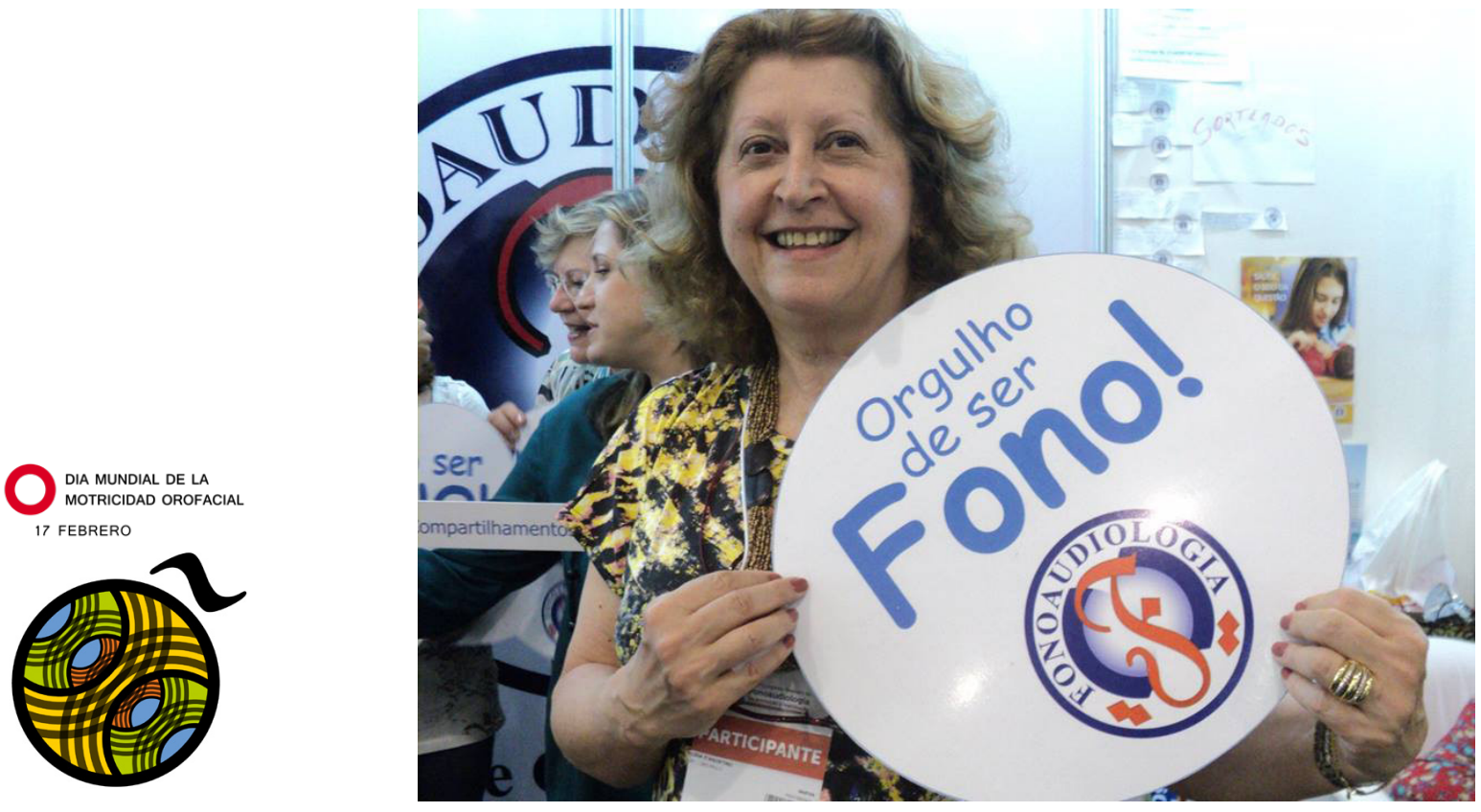